\title{
Can axillary lymph node ratio (LNR) predict local recurrences or deaths in patients with breast cancer with nodal involvement?
}

\author{
Bansal GJ*, Sophie Bright \\ The Breast Centre, Llandough University Hospital, Cardiff and Vale University Health Board, Penarth, United Kingdom
}

\begin{abstract}
Background: Axillary LNR (Lymph Node Ratio) is increasingly been contended as an important prognostic factor in breast cancer with varying results. The purpose of this study was to evaluate LNR with respect to both local recurrences and deaths.

Objective: To examine the value of the Lymph Node Ratio (LNR) in predicting local recurrence and death in breast cancer patients with lymph nodal involvement.

Methods: This is a retrospective study on 150 patients treated for node-positive breast cancer in the University health board between 2013 and 2014. The LNR was defined as the number of positive Lymph Nodes (LNs) over the total number of LNs removed. The LNR-risk groups were defined as low-risk, 0.01-0.2; intermediate-risk, $0.21-0.65$ and high- risk $L N R>0.65$.

Results: The mean follow-up time was 3.6 y and mean age of diagnosis was $57 \mathrm{y}$. Moderate- and highrisk LNR was significantly associated with $\mathrm{pN} 2$ and $\mathrm{pN} 3$ classification, pT3 classification and grade 2 tumours compared to low-risk LNR. There were a total of $9(6 \%)$ breast cancer local recurrences and 17 (11.3\%) breast cancer deaths; grouped together as 26 (17.3\%) 'Incidents' (recurrences/deaths). We found no significant difference between the incident rate and LNR group, pT classification, pN classification or age.

Conclusion: Higher LNR-risk groups were associated with higher $\mathrm{pN}$ and pT classification; however LNR was not a good indicator of breast cancer recurrence and death.
\end{abstract}

Keywords: Breast, Cancer, Axilla, Recurrence, Lymph nodes.

Accepted on July 20, 2018

\section{Introduction}

Breast cancer is the most common cancer in the UK and one of the most significant prognostic markers for breast cancer is axillary lymph node (LN) status, as an increase in the positive lymph node number is independently associated with poor clinical outcomes and increased recurrence rate [1]. Clinical practice has now changed from full dissection of the axilla to the use of Sentinel Node Biopsy (SNB), which determines the patients at high risk of nodal involvement who then require Axillary Lymph Node Dissection (ALND) [2]. The American Joint Committee on Cancer (AJCC) stages patients based on number of positive nodes found on dissection $(\mathrm{pN})$; stage $\mathrm{pN} 1$ if 1 to 3 nodes are involved, $\mathrm{pN} 2$ if $4-9$ and $\mathrm{pN} 3$ if $10+$ nodes are involved [3]. However, we now know that the number of positive lymph nodes recorded is partly influenced by the total number of lymph nodes harvested and examined [4,5] and previous studies have shown that patients may be under staged if their ALNDs are inadequate, leading to higher recurrence rates [6-11]. The current TNM classification system also uses the absolute number of lymph nodes involved, assuming that all dissections are the same [12]. In reality, the extent of axillary dissections varies hugely between countries, centres and surgeons, and therefore researchers are challenging the current classification systems and questioning whether more information on the number of nodes excised may be useful prognostically, helping to individualise treatment for patients more appropriately [13].

Recent studies explore the impact of the Lymph Node Ratio (LNR), defined as the number of positive LNs over the number of excised nodes [14-17]. There is evidence that LNR is a useful predictor of breast cancer recurrence and survival, and some studies found its prognostic superiority over absolute number of positive nodes [2,13,14,16,18-20]. This poses the question whether LNR should be considered in combination with the $\mathrm{pN}$ staging to guide decisions for breast cancer management.

The current study aims to examine the prognostic value of LNR, and specifically to evaluate whether LNR stratification leads to significantly different recurrence and mortality rates.

\section{Methods}

\section{Study population}

This is a retrospective study using data from the University Hospital database of breast cancer patients with a 3 to 4 year 
follow-up period. Ethical approval was waived due to the retrospective nature of the study. The population studied included 150 patients with invasive breast cancer who had original diagnosis in the Health board, between January, 2013 and October, 2014. Only patients with positive Sentinel Node Biopsies (SNBs) and subsequent Axillary Nodal Clearance (ANC) or direct ANC were included.

\section{Data analysis}

Data regarding local recurrence and death of patients was obtained using the hospital clinical portal system. 'Incidents' was defined as local recurrence or death. Local recurrence was defined as the recurrence of cancer in breast, regional lymph nodes and chest wall, ipsilateral to the primary tumour. Followup time was calculated from the date of diagnosis to the date of last follow-up or death (range: 3-4 y).

The LNR was calculated as the total number of positive lymph nodes divided by the total number of lymph nodes found and examined. The cohort was then divided into 3 groups based on established LNR cut-offs $[4,12,15,16,21]$ defined as low risk, 0.01-0.20; intermediate-risk, 0.21-0.65; and high-risk, $>0.65$. We examined the prognostic value of Lymph Node Ration (LNR) for patients with node-positive breast cancer with varying numbers of minimum nodes removed. We also examined the impact of minimum number of lymph nodes removed (all patients, $\leq 10,>10$ ) to compare the performance of LNR as prognostic indicator.

Comparison of categories within a characteristic was carried out with the Pearson Chi-square test and, if any of the expected frequencies was less than five, the Fisher exact test was used. The effect of LNR on incidents was investigated using bivariate logistical regression, using $p$ values of $<0.05$ as significant. Bivariate logistical regression was also used to analyse the effect of $\mathrm{pT}, \mathrm{pN}$ and age on recurrence and survival.

All statistical analyses were performed using SPSS statistical software (IBM SPSS statistics version 23).

\section{Results}

The mean age of diagnosis in our sample was 57 y (range, 26-90 y). Patients were diagnosed in 2013 and 2014 ( $n=93$ $(62 \%)$ and 57 (38\%) respectively) with follow-up time of 4 or 3 years respectively. Figure 1 shows the percentage of patients in each pN classification; $105(70 \%)$ in $\mathrm{pN} 1,26(18 \%)$ in $\mathrm{pN} 2$ and $12(8 \%)$ in pN3. Figure 2 shows the percentage of patients in each pT classification; 1 (1\%) in pT0, $33(22 \%)$ in pT1, 77 $(51 \%)$ in pT2, $26(17.3 \%)$ in pT3 and $4(3 \%)$ in pT4. Figure 3 shows the percentage of patients in each LNR classification; 86 $(57 \%)$ in low-risk group (LNR $\leq 0.2), 44(29 \%)$ in intermediate-risk group (LNR $>0.2$ and $\leq 0.65)$ and $20(13 \%)$ in high-risk group $(>0.65)$.

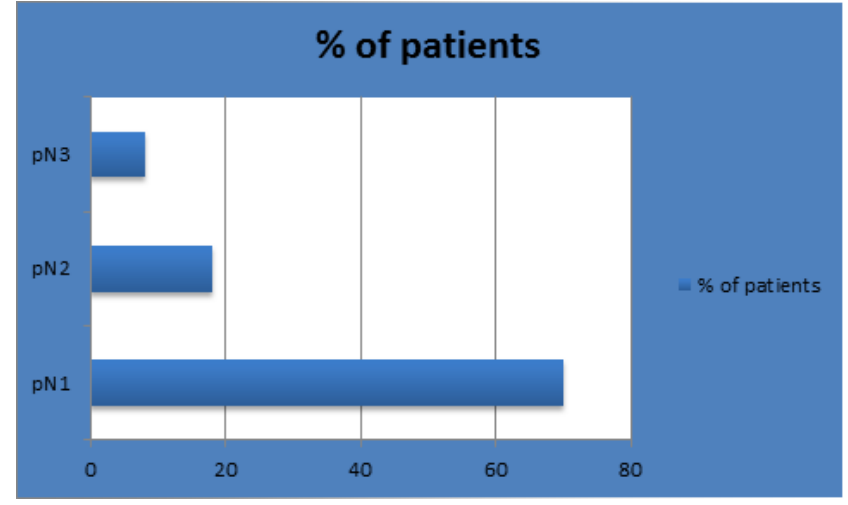

Figure 1. Distribution of $p N$ categories across the study group.

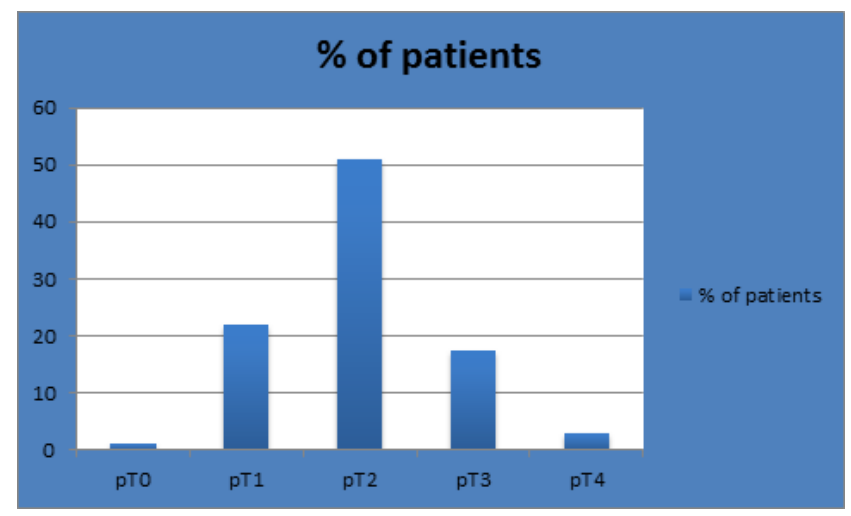

Figure 2. Distribution of pT categories across the study group.

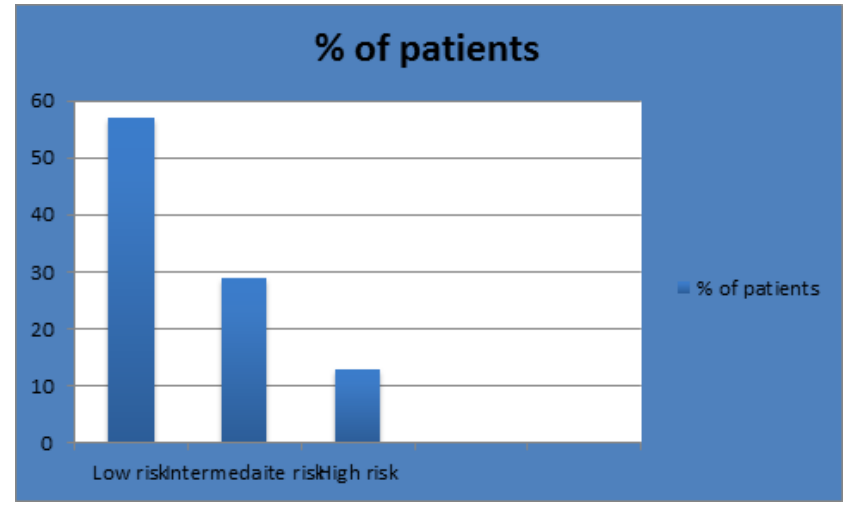

Figure 3. Distribution of LNR categories across the study group.

The clinical and pathological characteristics of patients according to LNR are shown in Table 1. Moderate- and highrisk LNR was significantly associated with $\mathrm{pN} 2$ and $\mathrm{pN} 3$ disease compared to low-risk LNR $(p=0.017)$. They were also significantly associated with grade 2 cancer $(p=0.000)$. Similarly, high-risk LNR, when compared to intermediate-risk were significantly associated with $\mathrm{pN} 3$ disease compared to $\mathrm{pN} 1$ disease $(\mathrm{p}=0.000)$. Lastly, high- and intermediate-risk LNR was significantly associated with pT3 disease (all p= 0.001). The relationship with age or other tumour 
Can axillary lymph node ratio (LNR) predict local recurrences or deaths in patients with breast cancer with nodal involvement?

characteristics did not vary significantly by LNR groups $(\mathrm{p}>0.05)$ (Table 1).

Table 1. Clinicopathologic characteristics of 150 women with lymph node-positive breast cancer according to lymph node ratio.

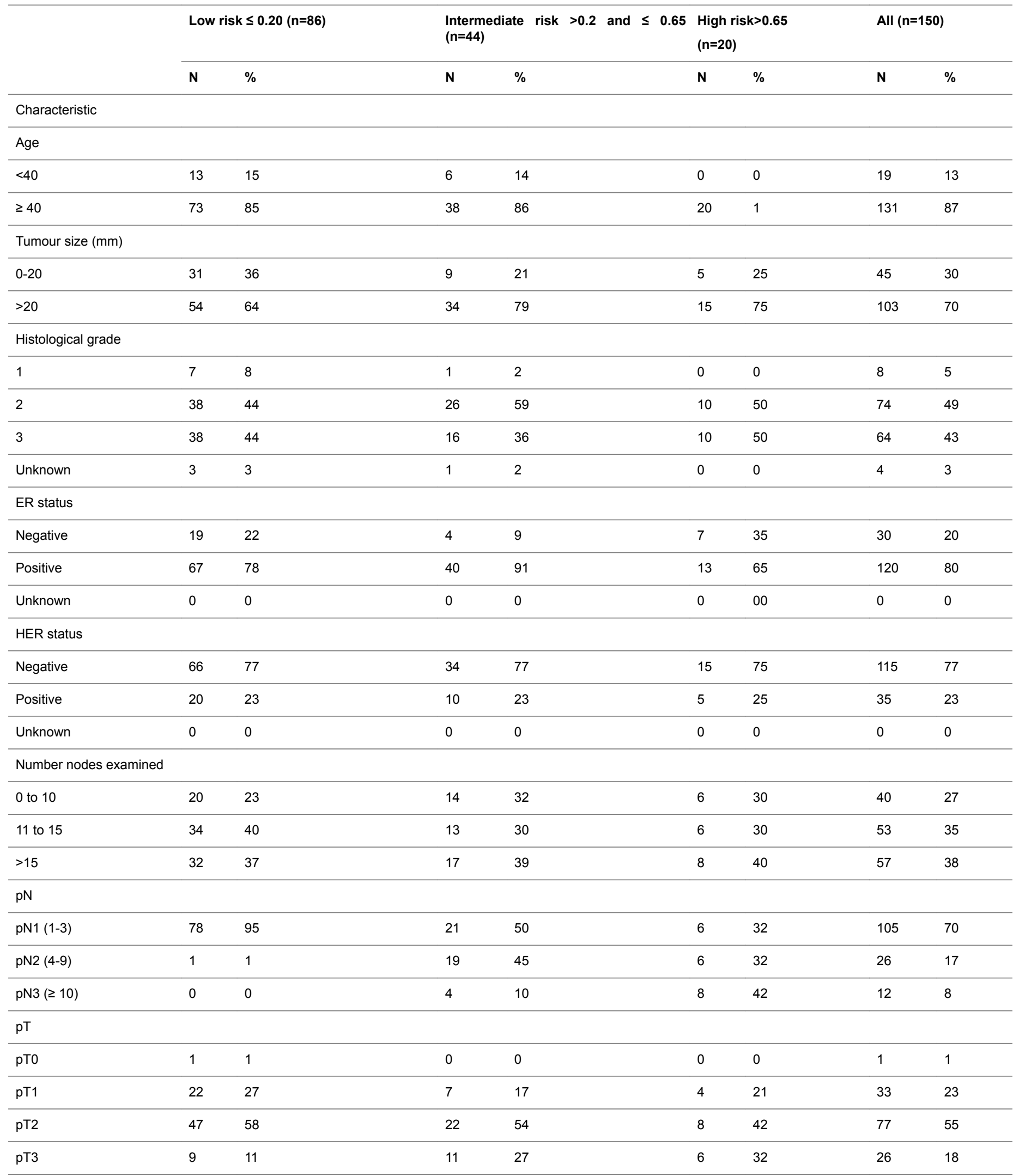




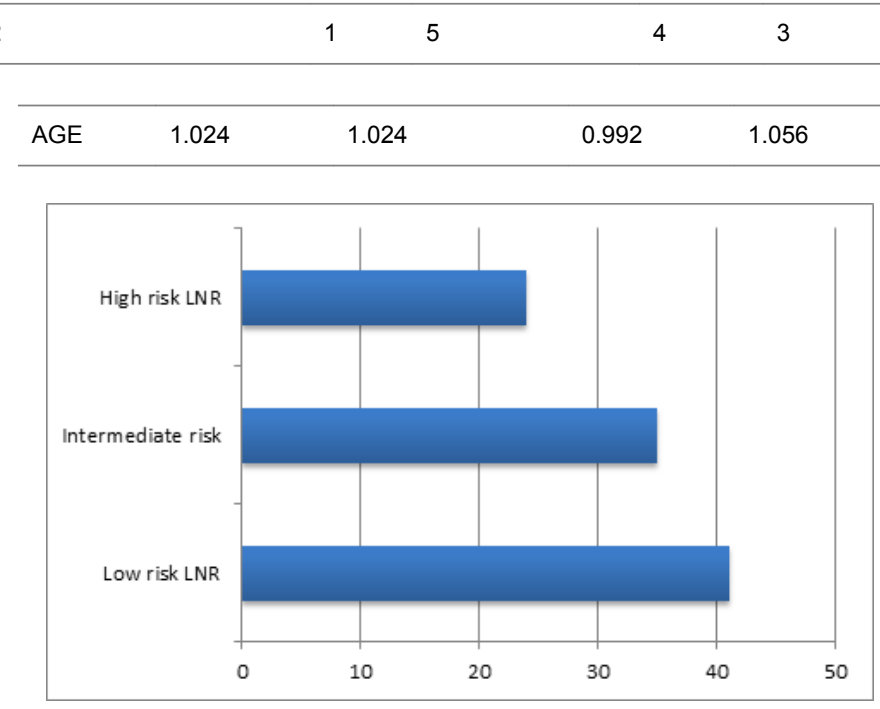

Figure 4. Percentages of incidents across the three LNR groups.

Table 3 shows the percentage breast cancer incidents by LNR and $\mathrm{pN}$ stage for varying number of nodes removed for 135 patients with available data. The mean number of nodes examined was 15 (median=14). There was no association between the number of LNs removed and incidents in each group $(p>0.05)$. There were higher incidents of recurrence or deaths in the high-risk LNR categories, specifically in the $\mathrm{pN} 2$ category and $<10$ nodes removed, but this did not reach statistical significance.

Table 3. Percent of incidents with respect to LNR and number of nodes dissected.

\begin{tabular}{|c|c|c|c|c|c|c|c|c|c|c|c|c|c|}
\hline \multirow[t]{2}{*}{ pN stage } & \multirow[t]{2}{*}{ LN's removed } & \multicolumn{3}{|c|}{$\begin{array}{l}\text { Low risk }(\leq 0.2) \\
(n=74)\end{array}$} & \multicolumn{3}{|c|}{$\begin{array}{l}\text { Intermediate risk }(>0.2 \text { and } \leq \\
0.65)(n=42)\end{array}$} & \multicolumn{3}{|c|}{ High risk $(>0.65)(n=19)$} & \multicolumn{3}{|l|}{ All $(n=135)$} \\
\hline & & $\begin{array}{l}\text { No of } \\
\text { incidents }\end{array}$ & Incidents & Total & $\begin{array}{l}\text { No of } \\
\text { incidents }\end{array}$ & Incidents & Total & $\begin{array}{l}\text { No of } \\
\text { incidents }\end{array}$ & Incidents & Total & $\begin{array}{l}\text { No of } \\
\text { incidents }\end{array}$ & Incidents & Total \\
\hline \multirow[t]{2}{*}{ pN1 } & $\leq 10$ & 0.00 & 17.00 & 0.00 & 0.00 & 1000 & 0 & 1.00 & 4.00 & 0.25 & 1.00 & 31.00 & 0.03 \\
\hline & $>10$ & 900 & 56.00 & 0.16 & 1.00 & 1000 & 0.10 & 1.00 & 2.00 & 0.50 & 1100 & 68. & 0.16 \\
\hline \multirow[t]{2}{*}{ pN2 } & $\leq 10$ & 0.00 & 1.00 & 0.00 & 200 & 400 & 50 & 1.00 & 2.00 & 0.50 & 3.00 & 7.00 & 43 \\
\hline & $>10$ & 0.00 & 0.00 & 0.00 & 3.00 & 14.00 & 0.21 & 0.00 & 4.00 & 0.00 & 3.00 & 18.00 & 17 \\
\hline \multirow[t]{2}{*}{ pN3 } & $\leq 10$ & 0.00 & 0.00 & 0.00 & 0.00 & 0.00 & 0.00 & 3 & 0.00 & 0.00 & 0.00 & 0.00 & 0 \\
\hline & $>10$ & 0.00 & 0.00 & 0.00 & 1.00 & 140 & 0.33 & 100 & 7.00 & 0.14 & 2.00 & 10.00 & 20 \\
\hline
\end{tabular}

\section{Discussion}

Tumour specific markers, such as ER and HER-2 status, are important prognostic indicators in breast cancer, but the presence and extent of axillary Lymph Node (LN) metastases remains most useful for prognostic assessment. However, studies show that the optimal method of classifying LN status remains to be elucidated. Current nodal staging systems solely use the number of positive axillary LNs, and although this parameter has been shown to be of value, recent literature queries whether additional information on the total number of LNs removed would be of prognostic benefit [13]. This is because data has shown that the chance of finding positive nodes increases with the number of LNs examined, and that under-staging of the axilla can be a result of inadequate dissections [2]. The concept of assessing LN status by ratios was first suggested years ago; a few parameters were developed but the lymph node ratio (LNR) was deemed the most clinically suitable [22]. Several studies have shown that LNR is more valuable than absolute positive LNs $(\mathrm{pN})$ in determining prognosis in breast cancer $[4,12,13,21]$ and many other cancers such as lung [23], gastric [24] and colon [25]. LNR has been proposed as a criterion to be incorporated in the revised breast cancer staging [26]. However, a definite cut-off 
value of LNR to predict long term outcome has not been established yet, despite multiple studies published on this subject.

In this study we showed that the higher the LNR the more likely the patient is to have a high $\mathrm{pN}$ classification. Higher LNR is also associated with high pT classifications and higher grade disease. However, there was no significant difference between the number of nodes examined and LNR group, neither was there an association between the LNR group and any of the following: HER- or ER-status, tumour size or age. Contrary to recent data, we found no significant difference between the incidents (local recurrence or death) and the LNR, even when each LNR-risk group was divided into two categories; $\leq 10$ and $>10$ nodes dissected. This suggests that the LNR is not an accurate indicator of chance of recurrence or death.

The results in this study are similar to Grassadonia et al. [27] in which the authors included LNR among the variables as prognostic factors in a cohort of patients with $\mathrm{pN} 3$ breast cancer. The authors used 0.6 as cut off and found that LNR did not influence prognosis. Within the limits of a retrospective study with small sample size, the authors concluded that once more than 10 lymph nodes are involved, LNR fails to maintain prognostic value. In a similar study by Schiffman et al. [6] LNR more than 0.65 was predictive of poor prognosis. This is similar to the cut off value of high-risk LNR used in our study. Their cohort included all patients ( $\mathrm{pN} 1, \mathrm{pN} 2$ and $\mathrm{pN} 3)$, but subgroup analysis on $\mathrm{pN} 3$ group revealed no difference in overall survival.

Another study [28] focussed on patients with stage 3 breast cancer and suggested 0.6 as the most sensitive LNR cut-off to detect the greatest difference in overall survival. Woodward et al. [18] carried out a large systematic review in 2006 that included over 32,000 patients from 24 studies between 1994 and 2005. This showed that overall LNR was a more accurate prognostic parameter than the absolute number of involved lymph nodes $(\mathrm{pN})$ in breast cancer. More recent studies also support this theory, showing that LNR is a superior predictor of survival than $\mathrm{pN}[4,6,12,13,21]$. Jayasinghe et al. supports the above statements when the data is grouped together, however the study went on to examine the prognostic value of LNR in breast cancer with patients divided into varying number of minimum nodes removed $(>5,>10>15)$. They found that LNR lost its significance in patients with $>10$ and $>15$ nodes dissected, perhaps suggesting that LNR holds value only when axillary dissections are inadequate. They concluded that oncologist can be confident that prognosis and decisions on adjuvant therapies can be based on $\mathrm{pN}$ classification alone when adequate axillary dissections are performed ( $>15$ nodes) but suggest that $\mathrm{pN}$ and LNR should be used on conjunction otherwise [2]. In our study, the mean number of axillary lymph nodes that were examined was [15], which could account for non-significance of our findings.

Modified LNR (mLNR) is a new parameter being explored in breast cancer patients. It is defined as $(\mathrm{pLN}+0.5) /(\mathrm{tLN}+0.5)$, where $\mathrm{pLN}$ is the number of positive LNs and tLN is the total number of nodes excised. The modifications to the standard LNR (adding 0.5 to both the numerator and denominator) were carried out to avoid results of zero in patients with nodenegative breast cancer. A 2017 study [22] using this parameter found it was useful in predicting the clinical outcomes of breast cancer patients independently, and in conjunction with $\mathrm{pN}$ stage. It was also found that the significance of mLNR was highest when patients had less than 10 LNs dissected or had no LN metastases [22]. Our study may have benefited from using mLNR had we included node-negative patients in our sample.

A further classification model proposed in the literature is the $\log$ odds of metastatic lymph nodes (LODDS). This value is defined as $\log ((\mathrm{pN}+0.5] /(\mathrm{nN}+0.5))$, where $\mathrm{pN}$ is the number of positive LNs and $\mathrm{nN}$ is the number of negative LNs, with the latter value calculated by subtracting $\mathrm{pN}$ from the total number of retrieved nodes [29]. Indeed a study carried out in 2015 found this parameter was also an independent prognostic indicator in breast cancer. The authors suggested an inferior value of LNR when there is limited harvest of lymph nodes, at which point LODDS is a better prognostic indicator [30].

An advantage of our study was using previously established LNR risk categories rather than creating categories for our own sample data like the majority of similar studies. We also had a high mean number of nodes removed in our sample (mean=15, median=14). This may suggest fewer patients had inadequate ALND than other studies. However we found no association between number of nodes dissected and incidents. Our study was unique in using incidents as our outcome measure as opposed to most of the studies using simply overall or breastcancer specific survival. An incident encompasses not only breast-cancer specific deaths and deaths due to other causes, but also local (ipsilateral) recurrence and so we have measured both adverse outcomes as a whole. Moreover, we believe that 'all causes mortality' is a better measure of prognosis than breast cancer specific survival in breast cancer studies. Most previous studies results were based on five-year survival rates, which are influenced by lead-time bias and over diagnosis [31]. The correlation between survival rate and mortality is zero for most common solid tumours [32]. This was the reason for using 'all cause' mortality rates in our study, leading to more reliable results.

One of the main limitation of our study was our sample size was relatively small $(n=150)$ and follow-up period fairly short (3-4 y) and thus this may account for the insignificant findings in our study. However unpublished data from our centre suggests most recurrences occur within 5 years of diagnosis. Larger studies with longer follow up could shed further light on this crucial issue.

In conclusion, our study showed that although LNR was a predictor of higher $\mathrm{pN}$ and $\mathrm{pT}$ classification and higher grade of breast cancer, it was not a good predictor of local recurrence or breast cancer deaths. 


\section{Conflicts of Interest}

The authors declare no conflict of interest/ funding sponsors for this study.

\section{References}

1. Rosen PRG, Saigo S, Kinne DW, Hellman S. A long-term follow-up study of survival in stage I (T1N0M0) and stage II (T1N1M0) breast carcinoma. J Clin Oncol 1989; 7: 355-366.

2. Jayasinghe U, Pathmanathan W, Elder N, Boyages E. Prognostic value of the lymph node ratio for lymph-nodepositive breast cancer- is it just a denominator problem? Springer Plus 2015; 4: 121.

3. American Joint committee on Cancer AJCC. Cancer Staging Handbook (6th Edn.). New York: Springer Verlag 2010; 417-460.

4. Vinh-Hung V, Verkooijen HM, Fioretta G, NeyroudCaspar I, Rapiti E, Vlastos G, Deglise C, Usel M, Lutz JM, Bouchardy C. Lymph node ratio as an alternative to $\mathrm{pN}$ staging in node-positive breast cancer. J Clin Oncol 2009; 27: 1062-1068.

5. Wang F, He W, Qiu H, Wang X, Guo G, Chen X. Lymph node ration and $\mathrm{pN}$ staging show different superiority as prognostic predictors depending on the number of lymph nodes dissected in Chinese patients with luminal a breast cancer. Clin Breast Cancer 2012; 12: 404-411.

6. Schiffman SC, McMasters KM, Scoggins CR, Martin RC, Chagpar AB. Lymph node ratio: a proposed refinement of current axillary staging in breast cancer patients. J Am Coll Surg 2011; 213: 45-52.

7. Saha S, Farrar WB, Young DC, Ferrara JJ, Burak WE. Variation in axillary node dissection influences the degree of nodal involvement in breast cancer patients. J Surg Oncol 2000; 73: 134-137.

8. Schaapveld M, Otter R, de Vries EG, Fidler V, Grond JA, van der Graaf WT, de Vogel PL, Willemse PH. Variability in axillary lymph node dissection for breast cancer. J Surg Oncol 2004; 87: 4-12.

9. Goldhirsch A, Glick JH, Gelber RD, Senn HJ. Meeting highlights: International Consensus Panel on the Treatment of Primary Breast Cancer. J Nat Cancer Inst 1998; 90: 1601-1608.

10. The Steering Committee on Clinical Practice Guidelines for the Care and Treatment of Breast Cancer. CMAJ 1998; 158: 1-2.

11. Chagpar AB, Scoggins CR, Martin RC, Sahoo S, Carlson DJ, Laidley AL. Factors determining adequacy of axillary node dissection in breast cancer patients. Breast J 2007; 13: 233-237.

12. Chagpar AB, Camp RL, Rimm DL. Lymph node ratio should be considered for incorporation into staging for breast cancer. Ann Surg Oncol 2011; 18: 3143-3148.

13. Truong PT, Vinh-Hung V, Cserni G, Woodward WA, Patricia T, Vlastos G. The number of positive nodes and the ratio of positive to excised nodes are significant predictors of survival in women with micrometastatic node-positive breast cancer. Eur J Cancer 2008; 44: 1670-1677.

14. Voordeckers M, Vinh-Hung V, Van De Steen J, Lamote J, Storme G. The lymph node ratio as prognostic factor in node-positive breast cancer. Radiother Oncol 2004; 70: 225-230.

15. Vinh-Hung V, Joseph SA, Coutty N, Ly BH, Vlastos G, Nguyen NP. Age and axillary lymph node ratio in postmenopausal women with T1-T2 node positive breast cancer. Oncologist 2010; 15: 1050-1062.

16. van der Wal BC, Butzelaar RM, van der Meij S, Boermeester MA. Axillary lymph node ratio and total number of removed lymph nodes: predictors of survival in stage I and II breast cancer. Eur J Surg Oncol 2002; 28: 481-489.

17. Truong PT, Lesperance M, Li KH, MacFarlane R, Speers $\mathrm{CH}$, Chia S. Micrometastatic node-positive breast cancer: long-term outcomes and identification of high-risk subsets in a large population-based series. Ann Surg Oncol 2010; 17: 2138-2146.

18. Woodward WA, Vinh-Hung V, Ueno NT, Cheng YC, Royce M, Tai P, Vlastos G, Wallace AM, Hortobagyi GN, Nieto Y. Prognostic value of nodal ratios in node-positive breast cancer. J Clin Oncol 2006; 24: 2910-2916.

19. Vinh-Hung V, Verschraegen C, Promish DI, Cserni G, Van de Steene J, Tai P, Vlastos G, Voordeckers M, Storme G, Royce M. Ratios of involved nodes in early breast cancer. Breast Cancer Res 2004; 6: R680-688.

20. Truong PT, Berthelet EL, Kader J, Olivotto IA. The prognostic significance of the percentage of positive/ dissected axillary lymph nodes in breast cancer recurrence and survival in patients with one to three positive axillary lymph nodes. Cancer 2005; 103: 2006-2014.

21. Danko ME, Bennet KM, Zhai JM, Olson JA. Improved staging in node-positive breast cacner patients using lymph node ration: results in 1,788 patients with longterm follow up. J Am Coll Surg 2010; 210: 797-805.

22. Wen J, Yang Y, Liu P, Ye F, Tang H, Huang X, Zhong S, Xie $\mathrm{X}$. Development and validation of a nomogram for predicting survival on the base of modified lymph node ratio in breast cancer patients. Breast 2017; 33: 14-22.

23. Matsuguma H, Oki I, Nakahara R, Ohata N, Igarashi S, Mori K, Endo S, Yokoi K. Proposal of new nodal classifications for non-small-cell lung cancer based on the number and ratio of metastatic lymph nodes. Eur J Cardiothorac Surg 2012; 41: 19-24.

24. Tong LL, Gao P, Wang ZN, Song YX, Xu YY, Sun Z, Xing $\mathrm{CZ}$, Wang $\mathrm{X}, \mathrm{Xu} \mathrm{HM}$. Can lymph node ratio take the place of $\mathrm{pN}$ categories in the UICC/AJCC TNM classification system for colorectal cancer? Ann Surg Oncol 2011; 18: 2453-2460.

25. Greenberg R, Itah R, Ghinea R, Sacham-Shmueli E, Inbar $\mathrm{R}$, Avital S. Metastatic lymph node ration (LNR) as a prognostic variable in colorectal cacner patients 
Can axillary lymph node ratio (LNR) predict local recurrences or deaths in patients with breast cancer with nodal involvement?

undergoing laparoscopic resection. Tech Coloproctol 2011; 15: 273-279.

26. Chagpar AB, Camp RL, Rimm DL. Lymph node ratio should be considered for incorporation into staging for breast cancer. Ann Surg Oncol 2011; 18: 3143-3148.

27. Grassadonia A, Vici P, Gamucci T, Moscetti L, Pizzuti L, entuccia L. Long term outcomeof breast cancer patientswith pathological N3a lymph node stage. Breast 2017; 32: 79-86.

28. Zhu $\mathrm{C}, \mathrm{Wu} \mathrm{XZ}$. Proposal of new classification for stage III breast cancer on the number and ratio of metastatic lymph nodes. J Surg Oncol 2012; 106: 696-702.

29. Tez M, Altundag K. Log odds of metastatic lymph nodes (LODDS) or negative to positive lymph node ratio? Surgery 2017; 162: 461.

30. Chen LJ, Chung KP, Chang YJ, Chang YJ. Ratio and logodds of positive lymph nodes in breast cancer patients with mastectomy. Surg Oncol 2015; 24: 239-247.
31. Gigerenzer G, Wegwarth O. Five year survival rates can mislead. BMJ 2013; 346: f548.

32. Welch HG, Schwartz LM, Woloshin S. Are increasing 5year survival rates evidence of success against cancer? JAMA 2000; 283: 2975-2978.

\section{*Correspondence to}

Bansal GJ

The Breast Centre

Llandough University Hospital

Cardiff and Vale University Health Board

United Kingdom 